\title{
ORIENTAÇ.ÃO NEURO-RADIOLÓGICA NOS PROCESSOS EXPANSIVOS DA FOSSA POSTERIOR
}

\author{
Sergio Raupp * \\ Eliseu Paglioli **
}

No diagnóstico dos processos expansivos da fossa posterior os métodos neuro-radiológicos ocupam lugar de destaque.

Radiologia simples - Os processos cirúrgicos da fossa posterior se acompanham com freqüência de hipertensão intracraniana que, radiològicamente, se manifesta por diastase de suturas (nos jovens), alterações selares, impressões digitiformes e atrofias ósseas.

Com valor localizador, dois tipos de neoplasia acarretam alteraçōes radiológicas dignas de nota: a) neurinomas do acústico, nos quais a sindrome clínica associada a alterações ósseas (dilatação do meato acústico interno), permite o diagnóstico de segurança em mais de $50 \%$ dos casos, sendo o estudo radiológico feito mediante três incidências fundamentais (Stenvers, Towne, Hirtz); b) meningiomas que, em algumas situações, acarretam alterações de aspecto mais ou menos caracteristico (erosões ósseas, aumento localizado da vascularização óssea, hiperostoses ou espiculas e, eventualmente calcificações intratumorais).

Radiologia com métodos contrastados - Para abordar os diversos métodos e concluir quanto à sua utilidade e indicação, há necessidade de dividir a fossa posterior em duas regiōes - parte anterior e parte posterior - divididas por um plano que passa pelo assoalho do IV ventrículo e aqueduto de Sylvius. Para o diagnóstico dos processos expansivos posteriores, a visibilização do ventrículo rombencefálico e do aqueduto é fundamental; já nos processos sediados na parte anterior, deve-se ter atenção especial no estudo das cisternas que formam o espaço subaracnóideo da fossa posterior. Na exploração radiológica se procura colocar em evidência, mediante contrastes positivos e negativos, seja as estruturas vasculares (angiografia vértebro-basilar), seja o sistema ventricular isoladamente (pneumoventriculografia, iodoventriculografia) ou o sistema ventricular e espaço subaracnóideo (cisternografia, iodomielencefalografia).

Trabalho do Instituto de Neurocirurgia de Pôrto Alegre (Rio Grande do Sul): * Assistente encarregado da Secç̃o de Neuro-radiologia; *: Diretor, neurocirurgião. 
A pneumoventriculografia consiste na contrastação do sistema ventricular com ar e, no caso específico, estudo da forma e posição do IV ventrículo e do aqueduto de Sylvius. Este método tem sua maior indicação nos processos expansivos da porção posterior da fossa cerebelar nos quais há deslocamento ventral do sistema ventricular. Por outro lado, a lateralização, ou não, da estrutura contrastada informa sôbre a situação lateral ou mediana da expansão. Para êsse fim procede-se a pequena trepanação frontal ou occipital para punção ventricular e introdução do contraste; nos pacientes com fontanela ainda aberta é desnecessária a trepanação.

$\mathrm{Na}$ análise da situação do IV ventrículo e do aqueduto, algumas linhas e pontos são indicados, como sejam: ponto de Twining, situado no meio de uma linha que se estende do tubérculo selar à protuberância occipital inrerna e que deve coincidir com o meio do IV ventrículo (fig. 1); linha de Lysholm, ângulo de Sutton, menos precisos porém de valor em determinadas situações.

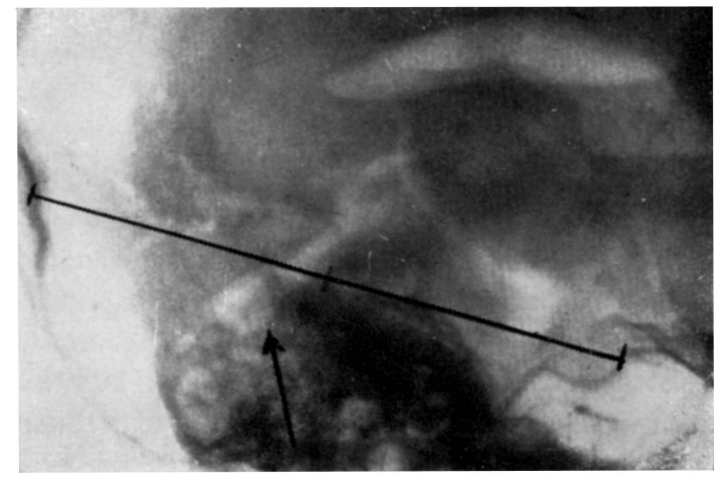

Fig. 1 - Ponto de Twin. ing (meio da linha entre a protuberância occipilal interna $e$ o tubérculo selar) que deve corresponder ao IV ventriculo. No caso há deslocamento dorsa' dêste ventriculo (tumor infiltrativo do tronco cerebral).

Se de um lado a situação baixa de um processo expansivo altera apenas o IV ventrículo em sua forma e posição, os processos altos alteram o ventrículo rombencefálico e o aqueduto ou sòmente êste último.

Os processos intraventriculares nem sempre modificam a situação ventricular, mas alteram sua forma amputando-lhe porçōes. Nunca é demais ter presente que o êrro diagnóstico de tumor infiltrante de tronco cerebral ao invés de tumor intraventricular afasta a possibilidade de intervenção cirúrgica.

Com freqüência podem surgir problemas quanto à origem, tumoral ou inflamatória, de uma estenose do aqueduto de Sylvius, utilizando-se, então, o método de Ruggiero e Castellano no qual são traçadas duas perpendiculares: uma paralela à apófise basilar, outra passando pela porção ânteroinferior do III ventrículo. Sempre que a parte posterior do ventrículo diencefálico mostrar-se levantada, será provável a existência de tumor na fossa posterior; sempre que o III ventrículo situar-se normalmente ou esteja abaixado será provável tratar-se de estenose inflamatória (fig. 2). 


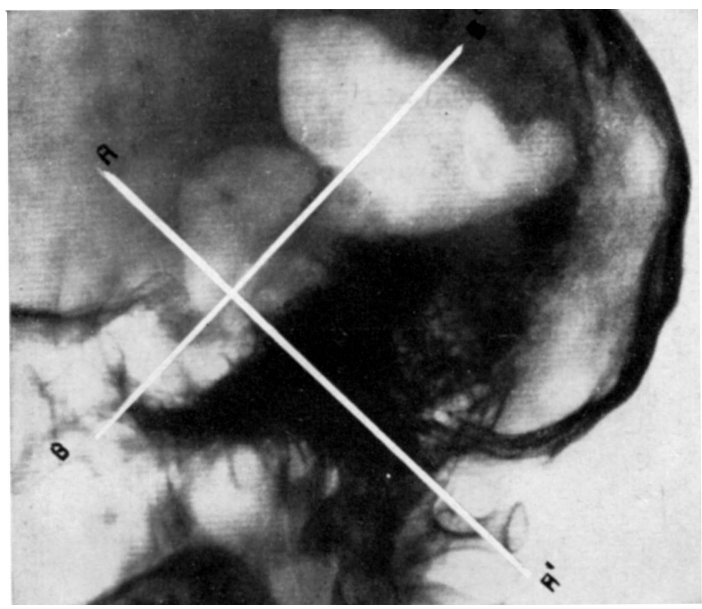

\begin{abstract}
Fig. 2 - Método de Ruggiero e Castellano: traca. se a linha AA' paralela à apófise basilar $e$, em sentido perpendicular, a linh. $B B^{\prime}$ que passa pela porçĩo ântero-inferior do III ventriculo. No caso a parte posterior do ventrículo diEncefálico não se mostr:x levantada lestenose pósinflamatória do aqueduto de Sylvius).
\end{abstract}

Nas aracnoidites da cisterna magna com fechamento dos orifícios de Luschka e Magendie, o IV ventriculo se apresenta dilatado, porém em situação, mediana e de forma simétrica (não confundir com a malformaçãa de Dandy-Walker).

Com freqüência as células da mastóide anormalmente desenvolvidas dificultam a visibilização do IV ventrículo. A tomada de clichês oblíquos ou a chamada autotomografia permite superar esta dificuldade: estando o paciente em decúbito ventral, com a região frontal apoiada sôbre a mesa radiológica, e tendo sido feito enchimento do IV ventriculo e do aqueduto de Sylvius com ar, solicita-se ao paciente que faça movimentos lentos basculando a cabeça, obtendo, ao mesmo tempo, clichês com raios horizontais (tempos longos de exposição).

Nos pacientes de pêso reduzido (crianças) pode-se, mediante manobras de duplo salto (Ziedzes des Plantes), visibilizar o sistema ventricular e também as cisternas anteriores da fossa cerebelar.

Alguns autores defendem o uso da ventriculografia central, com a colocação de uma sonda no III ventrículo, através do ventrículo lateral e orifício de Monro, com injeção ulterior de contraste negativo, o que permitiria a visibilização da parte posterior do III ventrículo, do aqueduto de Sylvius e IV ventriculo.

A iodoventriculografia tem como finalidade explorar as cavidades ventriculares com contraste positivo de tipo oleoso (Pantopaque, Lipiodol, Myodil). Alguns autores consideram êste método como rundamental para a investigação radiológica da fossa posterior. O contraste injetado no ventrículo lateral é dirigido, mediante contrôle radiológico, para o III ventrículo e, dai, para a fossa posterior. 
A nosso ver, à iodoventriculografia cabe papel de método suplementar à pneumoventriculografia ou à encefalografia gasosa, quando estas se mostrarem pouco satisfatórias. Isto ocorre em especial: em determinados casos de tumores intraventriculares e umbilicados no assoalho do IV ventrículo nos quais a introdução de ar nesta cavidade, seja por via lombar ou ventricular, acentua muito os vômitos, tornando impossivel a obtenção de bons clichês (fig. 3); em casos de tumores intraventriculares que aumen-
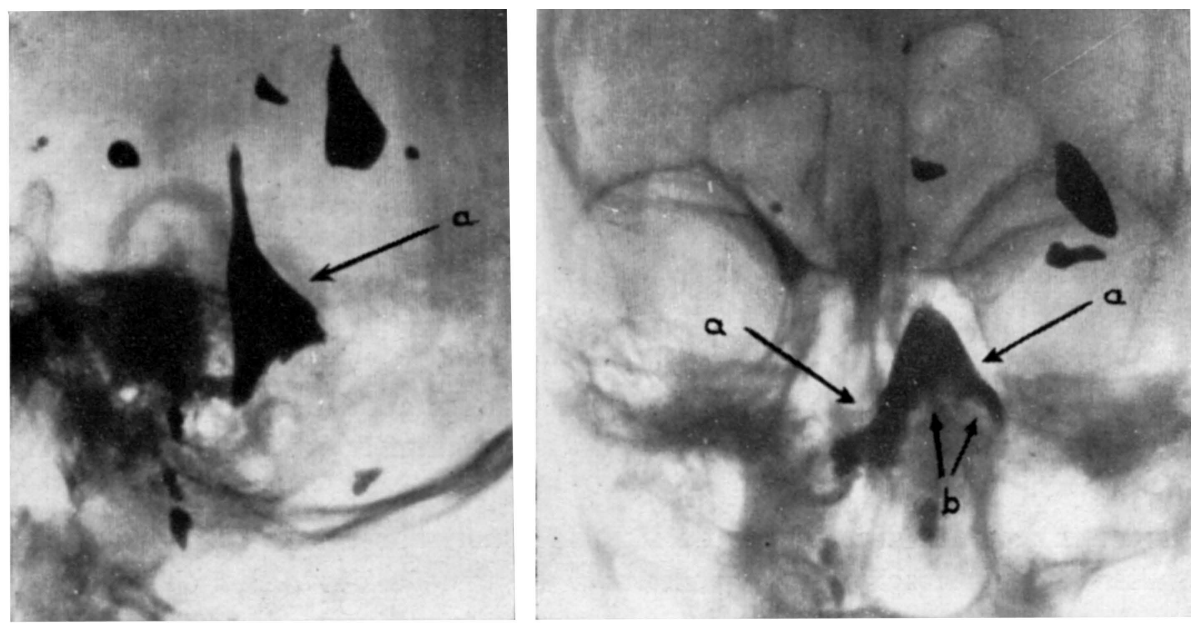

Fig. 3 - Iodoventriculografia: em perfil, o IV ventriculo mostra-se em posicão normal porém o clichê de face mostra amputação do recesso lateral esquerdo (b). Caso de ependimoma pediculado no assoalho do IV ventriculo, recesso lateral esquerdo.

tam e amputam de tal forma o IV ventriculo que a sua contrastação parcial pelo ar, não percebida, leva à suspeita de que se trata de tumor infiltrativo do tronco cerebral lateralizado, diagnóstico êste em desacôrdo com os dados clínicos (fig. 4); quando persiste dúvida em relação à etiologia — inflamatória ou tumoral - da estenose do aqueduto de Sylvius ou nas recidivas. Os argumentos contrários à iodoventriculografia seriam a lentidão ou não absorção dos contrastes com possiveis transtornos inflamatórios secundários (aracnoidites) e a determinação de alteraçōes anatômicas pós-inflamatórias que impossibilitam ulterior investigação com outros métodos.

Alguns autores injetam diretamente por sonda, no III ventriculo, o contraste positivo, denominando o método de iodoventriculografia central.

A encefalografia gasosa fracionada (EGF) consiste na injeção parcelada de pequenas quantidades de ar, orientadas para se dirigirem ora para o sistema ventricular ora para o espaço subaracnóideo, com a tomada concomitante de clichês em variadas posições, o que permite estudo seletivo da morfologia encefálica. 

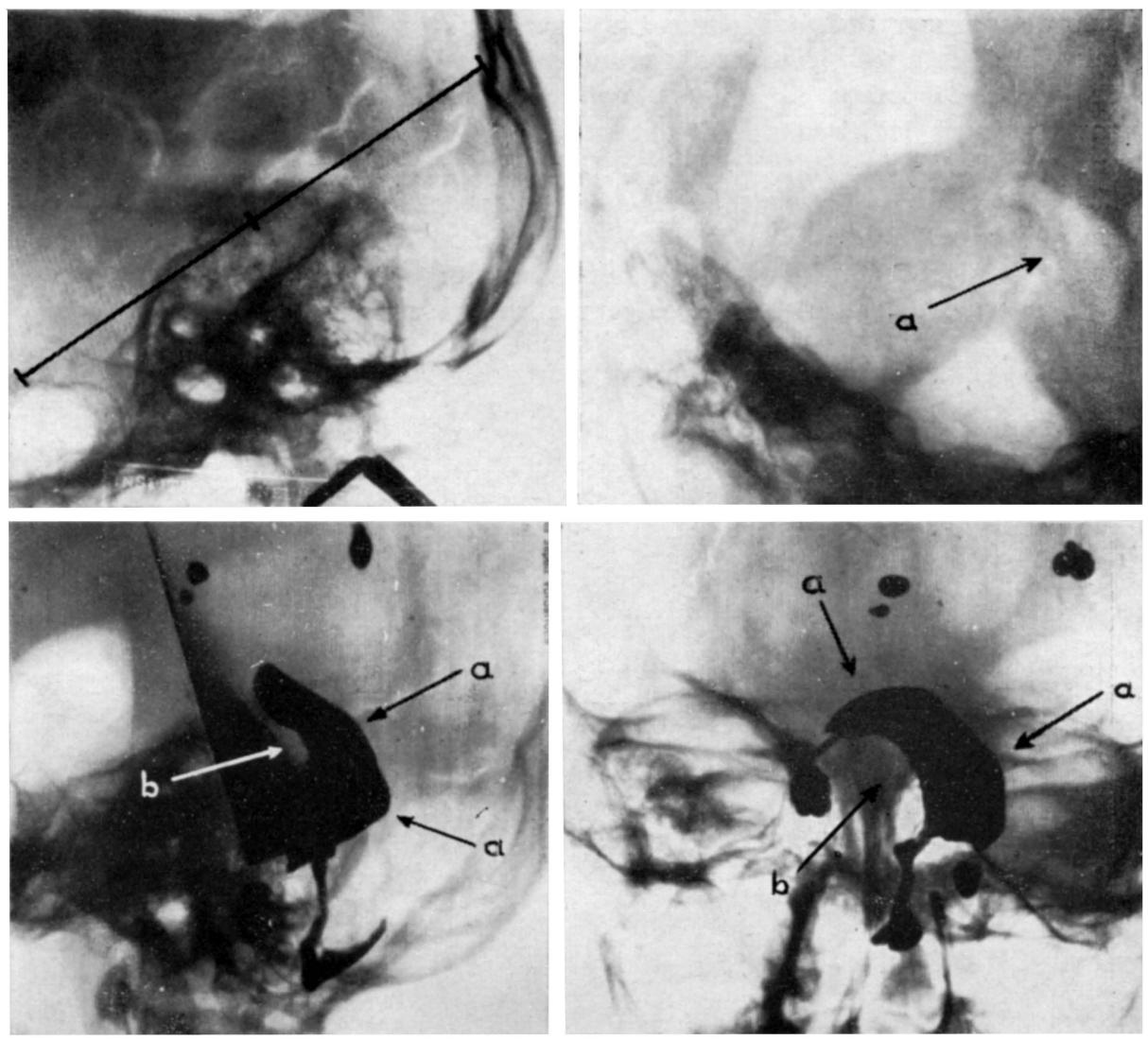

Fig. 4 - Na parte superior a encefalografia gasosa e a ventriculografia, respectivamente, mostram deslocamento dorsal (veja-se ponto de Twining) e para esquerda do IV ventriculo; na parte inferior a iodoventriculografia mostra situacão normal do ventrículo rombencefálico (a) que, entretanto, está dilatado e amputado (b) em seu recesso lateral direito. Diagnóstico cirúrgico: meduloblastoma do IV ventrículo, pediculado no teto da cavidade.

Nos processos da parte posterior, raramente se consegue a visibilização IV ventriculo e do aqueduto mediante a EGF: quando isto é obtido o diagnóstico pode ser feito por sinais diretos (fig. 5); caso contrário o diagnóstico é feito por sinais indiretos como sejam o achatamento da cisterna magna e o encravamento das amígdalas cerebelosas no buraco occipital (fig. 6); achatamento das cisternas pré-pontina e pré-peduncular (fig. 7); achatamento e elevação da cisterna de Galeno nos casos de hérnia transtentorial ascendente; aumento e distensão da cisterna do corpo caloso nos casos de hidrocefalia (fig. 7); visibilização da valécula do cerebelo deslocada lateralmente (fig. 8). $\dot{\mathrm{E}}$ interessante referir que o achatamento das cisternas magna, pré-pontina e pré-peduncular, assim como a herniação do cere- 
belo no buraco occipital podem ocorrer nos tumores supratentoriais; em tais casos a amputação da cisterna pré-peduncular permite o diagnóstico de localização exato. A encefalografia gasosa tem extraordinária utilidade nos processos localizados ventralmente ao IV ventriculo quer sejam intraencefálicos (gliomas infiltrantes do tronco cerebral, fig. 1) ou extraencefálicos (neurinomas do acústico, meningeomas do clivus, fig. 9). Nos casos em que as células da mastóide prejudicam a visibilização do IV ventrículo, usa-se a manobra descrita como autotomografia (ver pneumoventriculografia), estando o paciente sentado, com a cabeça antefletida.

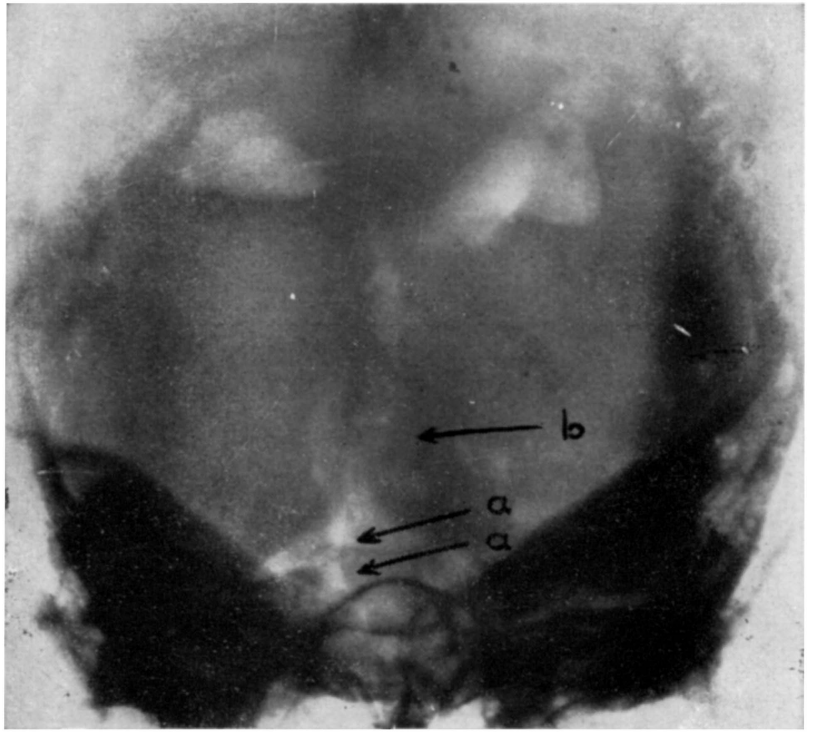

Fig. 5 - Desvio para a direita da valécula do cerebelo (a) e do IV ventriculo (b). Dragnóstico: meningioma da escama do occipital.

A cisternografia consiste na injeção de ar sòmente no espaço subaracnóideo, não parecendo ser satisfatório um método que estuda parcialmente a morfologia do encéfalo.

A iodomielencefalografia realiza-se pela injeção de contraste iodado no espaço subaracnóideo raqueano e ulterior drenagem do mesmo para as cisternas anteriores da fossa posterior, estando o paciente em decúbito ventral em mesa basculante. O método parece ser inferior aos outros até agora citados.

A angiografia é realizada pela injeção de contraste hidrossolúvel nas porções extracranianas dos vasos afluentes ao sistema vértebro-basilar. Das muitas técnicas preconizadas, as seguintes devem ser mencionadas: punção direta de uma das artérias vertebrais ao nivel do forame transversário; punção da artéria subclávia ou axilar, com injeção do contraste contra-corrente; cateterização da artéria vertebral via artéria radial ou femoral; panangiografia cerebral com injeção do contraste em uma das carótidas (Zaclis) . Quando interesse ùnicamente a porção intracraniana do sistema vértebro- 


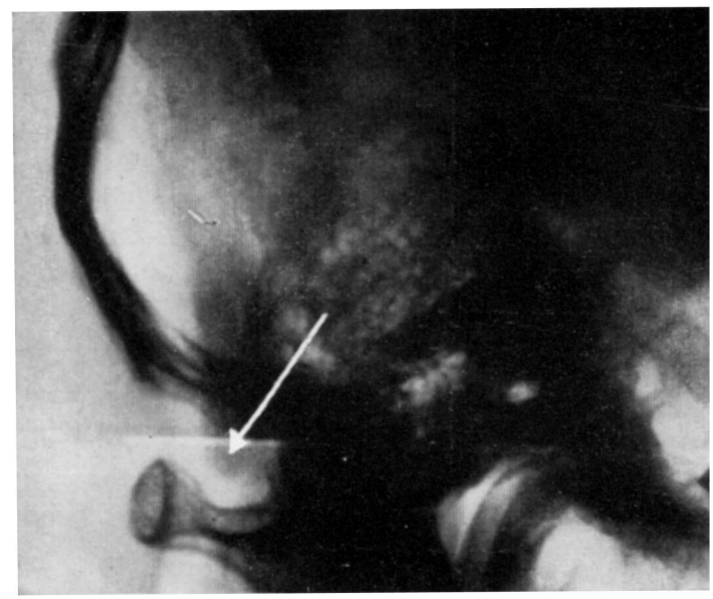

Fig. 6 - Imagem negativa das amigdalas cerebelares ultrapassando o buraco occipital, quase chegando ao nivel da primeira vértebra cervical. Diagnóstico: astrocitoma de verme cerebelar.

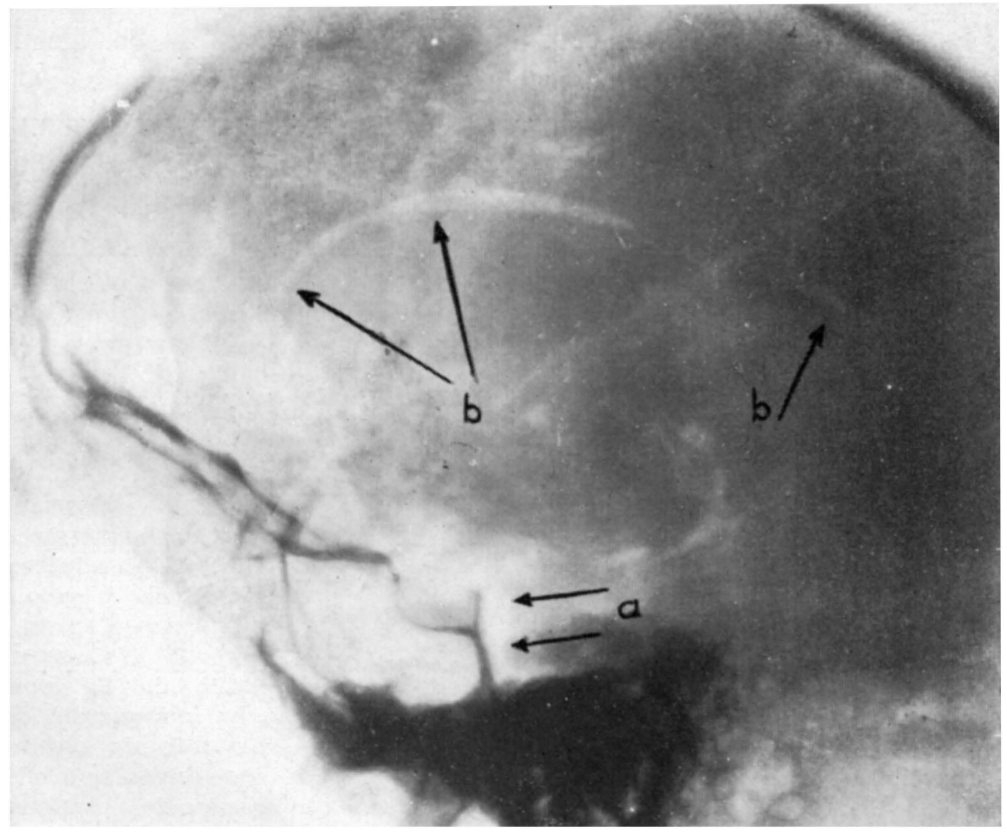

Fig. 7 - Achatamento das cisternas pré-pontina e pré-peduncular (a) $e$ distensão da cisterna do corpo caloso (b). Diagnóstico cirúrgico: meningioma incluso no hemisfério cerebeloso esquerdo. Nĭo foi descoberta a umbilicadura meningea. 


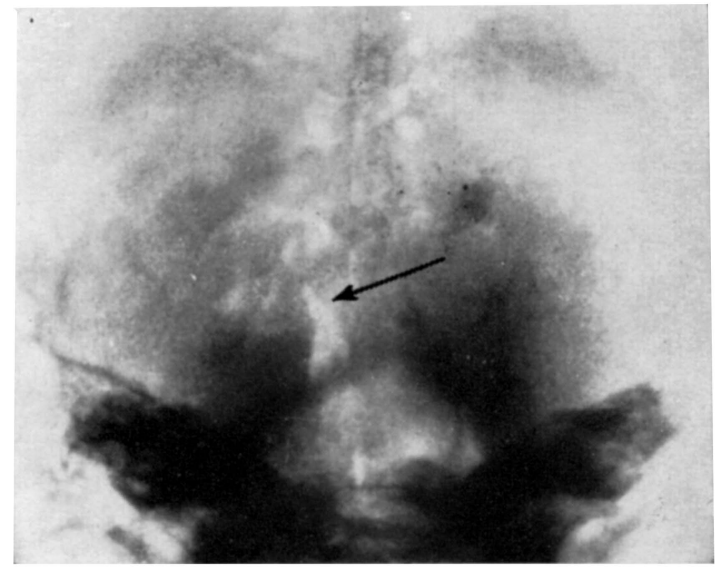

Fig. 8 - Mesmo caso da figura anterior. Observe-se o deslocamento da valécula do cerebelo para a direita. Ressalte-se como no presente caso a citada estrutura substitui o IV ventriculo na ausência de contrastação do mesmo.

basilar o método ideal é, sem dúvida, a punção direta da artéria vertebral; nos casos em que a artéria vertebral deva ser visibilizada em tôda a extensão optamos pela punção da artéria subclávia ou axilar, ou ainda, pelo cateterismo da artéria vertebral. A contribuição principal da angiografia vértebro-basilar é, sem dúvida, nos processos aneurismáticos e angiomatosos, assim como nos casos de tumores que permitem visibilização direta pela contrastação de sua arquitetura vascular (meningeomas, hemangiomas). Nos processos eypansivos da fossa posterior, a angiografia permite o diagnóstico de hipertensão (abaixamento da artéria cerebelosa póstero-inferior, levantamento da artéria cerebelosa superior, deslocamento ventral do tronco basiJar), sendo mais discreta sua contribuição quanto a dados de localização

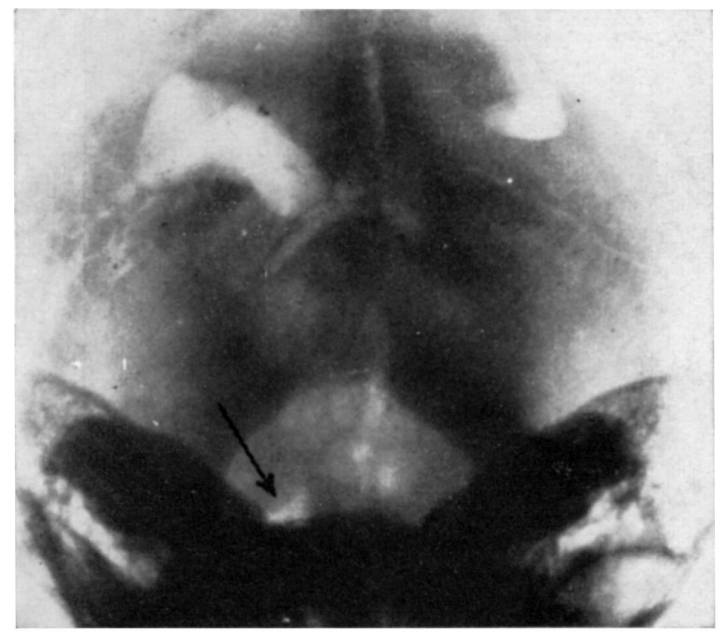

Fig. 9 - Observe-se que o ar na cisterna do ângulo pontocerebeloso áreito delimita o pólo medial de volumoso tumor extracerebral. Note-se ainda o desvio do IV ventrículo para a esquerda, além da dilatação da cisterna circunpeduncular direita, evidenciando a assimetria do pedúnculo em relaçõo ao buraco de Pacchioni. Diagnóstico: neurinoma de acústico direito. 
idesvio lateral do ramo medial da artéria cerebelosa póstero-inferior), ainda que nos últimos anos vários autores tenham codificado quadros angiográficos específicos.

Resta lembrar a contribuição da angiografia carotidea: ela pode mostrar sinais de dilatação ventricular, levando o radiologista a investigar o III ventrículo, o aqueduto e o IV ventrículo; em alguns casos de processos sediados na tenda do cerebelo (meningeomas, angiomas) a carótido-angiografia poderá mostrar a presença de vasos emergindo da porção intracavernosa do sifão carotídeo e que vascularizam a tenda e se apresentam hipertrofiados, não sendo visibilizados no angiograma normal (fig. 10).

Nos últimos anos tem sido pôsto em foco o emprêgo da tomografia $\epsilon \mathrm{m}$ neuro-radiologia.

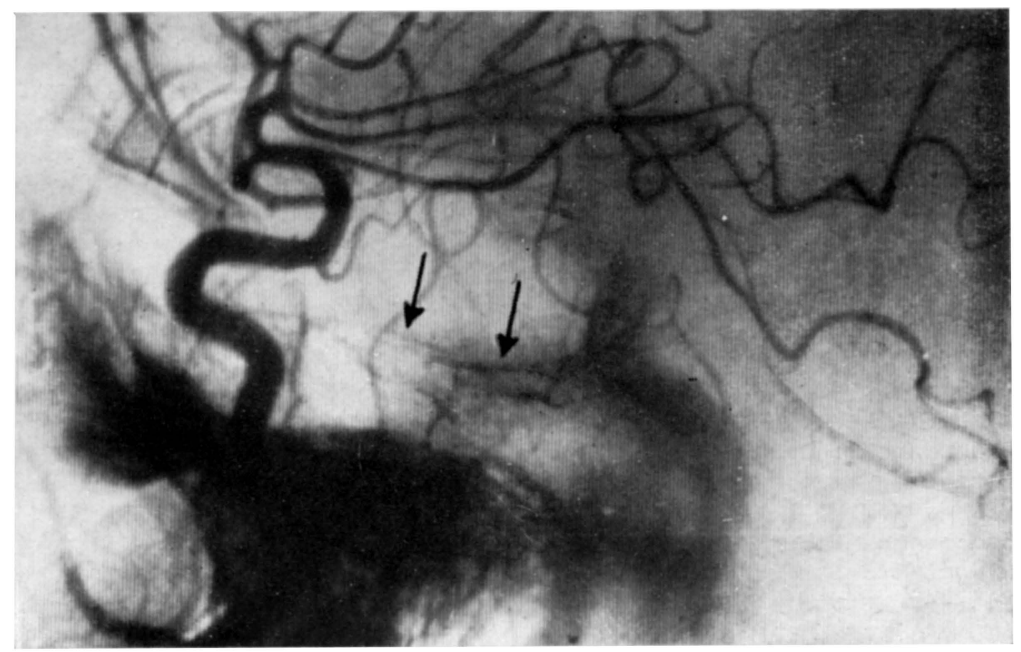

Fig. 10 - Angiograma da carótida primitiva direita, notando-se pequenos vasos meningeos, hipertrofiados, nascidos na porção intracavernosa do sifão carotideo e que vascularizam parcialmente neoplasia do cerebelo. Diagnóstico: meningioma da tenda do cerebelo.

\section{RESLMO}

Os autores revêem sua experiência e procuram traçar esquemàticamente sua orientação neuro-radiológica na pesquisa e diagnóstico dos processos expansivos da região cerebelosa. A pneumo e iodoventriculografia, a encefalografia gasosa e a angiografia vértebro-basilar são os exames de escolha, sendo apresentados casos ilustrativos. A mais exata conclusão anatômica é o que importa; os métodos são secundários. O manejo de tôdas as técnicas é fundamental. 


\section{SUMMARY}

\section{Neuro-radiologic orientation in the space-occupying processes of posterior fossa.}

The authors review their experience and establish a neuro-radiologic orientation in the investigation and diagnosis of the space-occupying processes of cerebelar region. The methods of choice are the pneumo and iodoventriculography, the gaseous encephalography and vertebral-basilar angiography. Illustrative cases are presented. The important aspect is the exact anatomical localization, while the methods are secondary. The carrying-our of all the techniques is fundamental.

\section{REFERENCIAS}

1. BULL, J. W. D. - Positive contrast ventriculography. Acta Radiol., 34:253, 1950. 2. CARRILlo, R. - Diagnóstico Yodoventriculográfico de los Tumores Infratentoriales. Imprenta Frascoli y Bindi, Buenos Aires, 1937. 3. DAVIDOFF, L. M. \& EPSTEIN, B. S. - The Abnormal Encephalogram. Lea \& Febiger, Philadelphia, 1950. 4. Di CHIRO, G. - An Atlas of Detailed Normal Pneumoencephalographic Anatomy. Charles C. Thomas, Springfield (Illinois) 1961. 5. DILENGE, D.; DAVID, M. \& TALAIRACH, J. - A propos des indications et de la technique de l'iodoventriculographie. Neuro-Chir., 6:347, 1960. $€$. HAUGE, T. - Catheter vertebral angiography. Acta Radiol., Supl. 109, 1954. 7. JOHANSON, C. - The central veins and deep dural sinuses of the brain. Acta Radiol., Supl. 107, 1954. 8. LILIEQUIST, B. - The anatomy of the sub-arachnoid cisterns. Acta Radiol., Supl. 46, 1956.9. LINDGREN, E. - Some aspects on the technique of encephalography. Acta Radiol., 31:161, 1949. 10. LINDGREN, E. - Percutaneous angiography of the vertebral artery. Acta Radiol., 33:331, 1950. 11. LINDGREN, E. - Encephalographic examination of tumours in the posterior fossa. Acta Radiol., 34:331, 1950. 12. LINDGREN, E. - Radiologic examination of the brain and spinal cord. Acta Radiol., Supl. 151, 1957. 13. LYSHOM, E.; EBENIUS, B.; LINDBLOM, K. \& SAHLSTED, H. - DaS Ventrikulogram. Acta Radiol., Supl. 24, 25 e 26, 1935-37. 14. PAGLIOLI, E. Ventriculografi.a. Editora Globo, Pôrto Alegre, 1938. 15. PORTUGAL, J. R. \& SAMPAIO, P. - Contribuição ao estudo anatômico e radiológico das artérias da fossa posterior. J. Bras. Neurol., 6:301, 1954. 16. RADNER, S. - Vertebral angiography by catheterization. Acta Radiol., Supl. 87, 1951. 17. RUGGIERO, G. - L'Encephalografie Fractionée. Masson et Cie., Paris, 1957. 18. SUTTON, D. - The radiological assesment of the normal aquaeduct and 4th ventricle. Brit. J. Radiol., 23:208, 1950. 19. TENUTO, R. A. - Iodoventriculografia. Arq. Neuro-Psiquiat. (São Paulo) Supl. 1, 1954. 20. TWINING, E. W. - Radiology of the third and fourth ventricles. Brit. J. Radiol., 12:385, 1939. 21. WICKBOM, I. - Angiography of the carotid artery. Acta Radiol., Supl. 72, 1948. 22. ZACLIS, J. - Visibilização radiográfica de todo o sistema vascular encefálico mediante injeção de contraste em uma única artéria: pan-angiografia cerebral. Arq. Neuro-Psiquiat. (São Paulo) 17:1, 1959.23. ZIEDSES DES PLANTES, B. G. - Examen du troisième et du quatrième ventricules au moyen de petites quantités d'air. Acta Radiol., 34:399, 1950.

Instituto de Neurocirurgia - Hospital São Francisco - Pôrto Alegre, Rio Grande do Sul-Brasil. 